Received: 2021.03.09 Accepted: 2021.07.27 Available online: 2021.08 .26 Published: 2021.10.02

Authors' Contribution: Study Design A Data Collection B Statistical Analysis $C$ Data Interpretation D Manuscript Preparation E Literature Search F Funds Collection G
ABEF 1,2 Shinsuke Takeda

AEF 3 Yoshihiro Tanaka

BE 4 Matsuyoshi Maeda

E 2 Hikaru Hayakawa

E 1,2 So Mitsuya

E 2 Ken-ichi Yamauchi
1 Trauma and Microsurgery Center, Toyohashi Municipal Hospital, Toyohashi, Aichi, Japan

2 Department of Orthopedic Surgery, Toyohashi Municipal Hospital, Toyohashi, Aichi, Japan

3 Department of Preventive Medicine, Northwestern University Feinberg School of Medicine, Chicago, IL, USA

4 Department of Pathology, Toyohashi Municipal Hospital, Toyohashi, Aichi, Japan
Corresponding Author: Financial support: Conflict of interest:
Shinsuke Takeda, e-mail: s7.takeda.jpn@gmail.com

None declared

None declared

\begin{abstract}
Patient: Male, 49-year-old
Final Diagnosis:

Symptoms:

Medication:

Clinical Procedure:

Specialty:

Objective:

Background:

Case Report:

Conclusions:

Chromobacterium violaceum infection

No symptom

$-$

Infectious Diseases

\section{Rare disease}

Chromobacterium violaceum (C. violaceum) is a gram-negative and facultative anaerobic oxidase-positive bacillus generally seen in tropical or subtropical areas (latitudes between $35^{\circ} \mathrm{N}$ and $35^{\circ} \mathrm{S}$ ). C. violaceum infection is a rare but serious infection with high morbidity and mortality rates. Most clinicians practicing in non-tropical counties, such as Japan, are unfamiliar with it.

We report the first fatal case of a 49-year-old man infected with $C$. violaceum after a traffic accident in Japan (latitude $34.8^{\circ} \mathrm{N}$ ). The patient reported brief submergence in a marshy muddy rice field after the accident. There was some evidence of soil and water contamination of the patient's skin and clothing, but he denied swallowing water or soil. There were no findings of pneumonitis or severe open wounds on admission. Until the night of the $7^{\text {th }}$ day of hospitalization, his general conditions remained stable despite a persistent fever. However, he suddenly collapsed on the $8^{\text {th }}$ day of hospitalization and died. C. violaceum bacteremia led to fatal sepsis on dissemination to the iliopsoas abscess, which is a rare combination for this infection.

Episodes of exposure to contaminated water or soil, especially in summer, are important predisposing factors for $C$. violaceum infection. Thus, it is vital to include $C$. violaceum infections as a differential diagnosis, since the mortality rate of $C$. violaceum infections is high and the cases of this infection have increased in non-tropical counties.
\end{abstract}

Keywords:

\section{Chromobacterium violaceum • Fatal Outcome • Psoas Abscess}

Full-text PDF: https://www.amjcaserep.com/abstract/index/idArt/932037

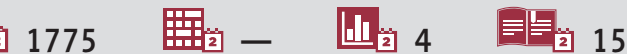




\section{Background}

Chromobacterium violaceum (C. violaceum) is a gram-negative, facultative anaerobic oxidase-positive bacillus [1-3]. As $C$. violaceum is endemic in natural aquatic environments and is temperature sensitive, it has a predilection for tropical and subtropical areas (latitudes between $35^{\circ} \mathrm{N}$ and $35^{\circ} \mathrm{S}$ ) [1,2]. $\mathrm{C}$. violaceum infections in humans usually occur after exposure to contaminated soil or water, following traumatic soft tissue injuries, or water aspiration after near-drowning episodes [1-4]. Infections generally occur in summer, in mostly young and healthy patients $[1,4,5]$. Most clinicians practicing in non-tropical countries such as Japan are likely to be unfamiliar with this rare infection [1]. Here, we describe the first fatal case of C. violaceum infection that occurred after a traffic accident.

\section{Case Report}

A 49-year-old man with a no significant past medical history had a vehicle accident (motorcycle-to-car collision) and fell into the rice paddies in Toyohashi City, Aichi Prefecture, Japan (latitude $34.8^{\circ} \mathrm{N}$ ), in August 2019. He was transferred to our Emergency Department with a high-energy trauma injury. The patient reported brief submergence in a marshy muddy rice field after the accident and denied swallowing water or soil. He also claimed to be conscious throughout the episode.

On admission, he reported feeling severe pain in his back, lumbar region, bilateral femur, and right thumb. There were no respiratory symptoms such as dyspnea. He denied any recent travel history, recent alcohol intake, illicit drugs, or regular medication intake. His vital signs on admission were as follows: Glasgow Coma Scale, E4V5M6; temperature, $36.3^{\circ} \mathrm{C}$; heart rate, $90 \mathrm{bpm}$; blood pressure, 141/63 $\mathrm{mmHg}$; respiratory rate, 18 breaths/min; and $100 \%$ oxygen saturation, on a $100 \%$ non-rebreather reservoir mask at $10 \mathrm{~L} / \mathrm{min}$ of oxygen. On physical examination, there was some evidence of soil and water contamination of the patient's skin and clothing. His speech and food intake were unaffected. Respiratory sounds were clear to auscultation bilaterally and there were no severe open wounds. Only slight abrasion on bilateral knees was observed.

Radiography showed fractures of the right trochanteric femur, left distal femur, bilateral scapula, right thumb, and right lumbar (L1-L4) transverse process, and sixth thoracic vertebral compression fracture. Computed tomography (CT) showed right pulmonary contusion, right rib fractures (ribs 10-12), and slight hemorrhage of the right psoas major muscle because of a right lumbar (L1-L4) transverse process fracture. There were no findings of pneumonitis (Figure 1). None of the fractures were open fractures. Blood test results revealed significantly elevated white blood cell (WBC) count of $15.3 \times 10^{3} / \mu \mathrm{L}$ with
$67.3 \%$ of neutrophils; hemoglobin $(\mathrm{Hb}), 12.7 \mathrm{~g} / \mathrm{dL}$; platelet (Plt), $18.8 \times 10^{4} / \mu \mathrm{L}$; C-reactive protein (CRP), $0.01 \mathrm{mg} / \mathrm{dL}$; total protein (TP), $6.3 \mathrm{~g} / \mathrm{dL}$; albumin (Alb) $3.9 \mathrm{~g} / \mathrm{dL}$; aspartate aminotransferase (AST), $213 \mathrm{U} / \mathrm{L}$; alanine aminotransferase (ALT), $151 \mathrm{U} / \mathrm{L}$; total bilirubin (T.bil) $0.6 \mathrm{mg} / \mathrm{dL}$; creatine kinase (CK), $490 \mathrm{U} / \mathrm{L}$; blood urea nitrogen (BUN), $15 \mathrm{mg} / \mathrm{dL}$; creatinine (Cre), $0.63 \mathrm{mg} / \mathrm{dL}$; prothrombin time-International normalized ratio (PT-INR), 0.97; fibrinogen (Fib), $233 \mathrm{mg} / \mathrm{dL}$. Electrocardiograms did not reveal any remarkable findings.

On admission, we administered nonsteroidal anti-inflammatory drugs (NSAIDs) for his pain. Both femoral fractures required standby operations. On the $4^{\text {th }}$ day of hospitalization, we performed surgery to repair his right trochanteric femoral fractures through open reduction and internal fixation under lumbar spinal anesthesia. On the $5^{\text {th }}$ day of hospitalization, his fever increased to almost $40^{\circ} \mathrm{C}$ (Figure 2), which was higher than the expected postoperative day 1 temperature. We also examined 2 sets of blood cultures. We treated the patient with a perioperative antibiotic, cefotiam, to prevent surgical site infection on the $4^{\text {th }}$ and $5^{\text {th }}$ days of hospitalization. Blood test results on the $5^{\text {th }}$ day revealed a WBC count of $7.46 \times 10^{3} / \mu \mathrm{L}$ with $74.1 \%$ neutrophils; Hb, $7.6 \mathrm{~g} / \mathrm{dL}$; Plt, 15.0×104/L; CRP, $19.95 \mathrm{mg} / \mathrm{dL}$; AST, 61 U/L; ALT, 60 U/L; CK, 1352 U/L; PT-INR, 1.06; Fib, 736 $\mathrm{mg} / \mathrm{dL}$. The patient also received a red blood cell transfusion $(280 \mathrm{~mL})$ on the $5^{\text {th }}$ day of hospitalization. His fever was below $38^{\circ} \mathrm{C}$ on the $6^{\text {th }}$ and $7^{\text {th }}$ days of hospitalization (Figure 2).

Until the night of the $7^{\text {th }}$ day of hospitalization, his back and bilateral femur pain persisted. However, his general conditions, including speech and food intake, remained stable, and he experienced no respiratory distress. There were no signs of surgical site infection or infected wounds. The patient experienced insomnia on the night of the $7^{\text {th }}$ day because of postoperative left femur pain. In the early morning of the $8^{\text {th }}$ day of hospitalization, chest pain gradually appeared in along with dyspnea and cyanosis. Acute coronary syndrome, acute aortic dissection, cardiac tamponade, and pulmonary embolism were suspected and we performed blood tests, 12-lead electrocardiography (ECG), and echocardiography and then prepared for contrast-enhanced CT. The ECG newly identified a left bundle branch block, suggesting a new onset of coronary artery disease. Echocardiography showed no pericardial effusion, no right ventricular (RV) enlargement, no RV free wall hypokinesis, and mild tricuspid valve regurgitation. These findings suggested that this patient had no cardiac tamponade and was less likely to have pulmonary embolism.

Before the patient underwent $\mathrm{CT}$, he went into shock and cardiopulmonary arrest shortly thereafter. Advanced cardiac life support was initiated according to the American Heart Association guidelines [6]. Percutaneous cardiopulmonary support (PCPS) was introduced immediately for cardiopulmonary resuscitation 


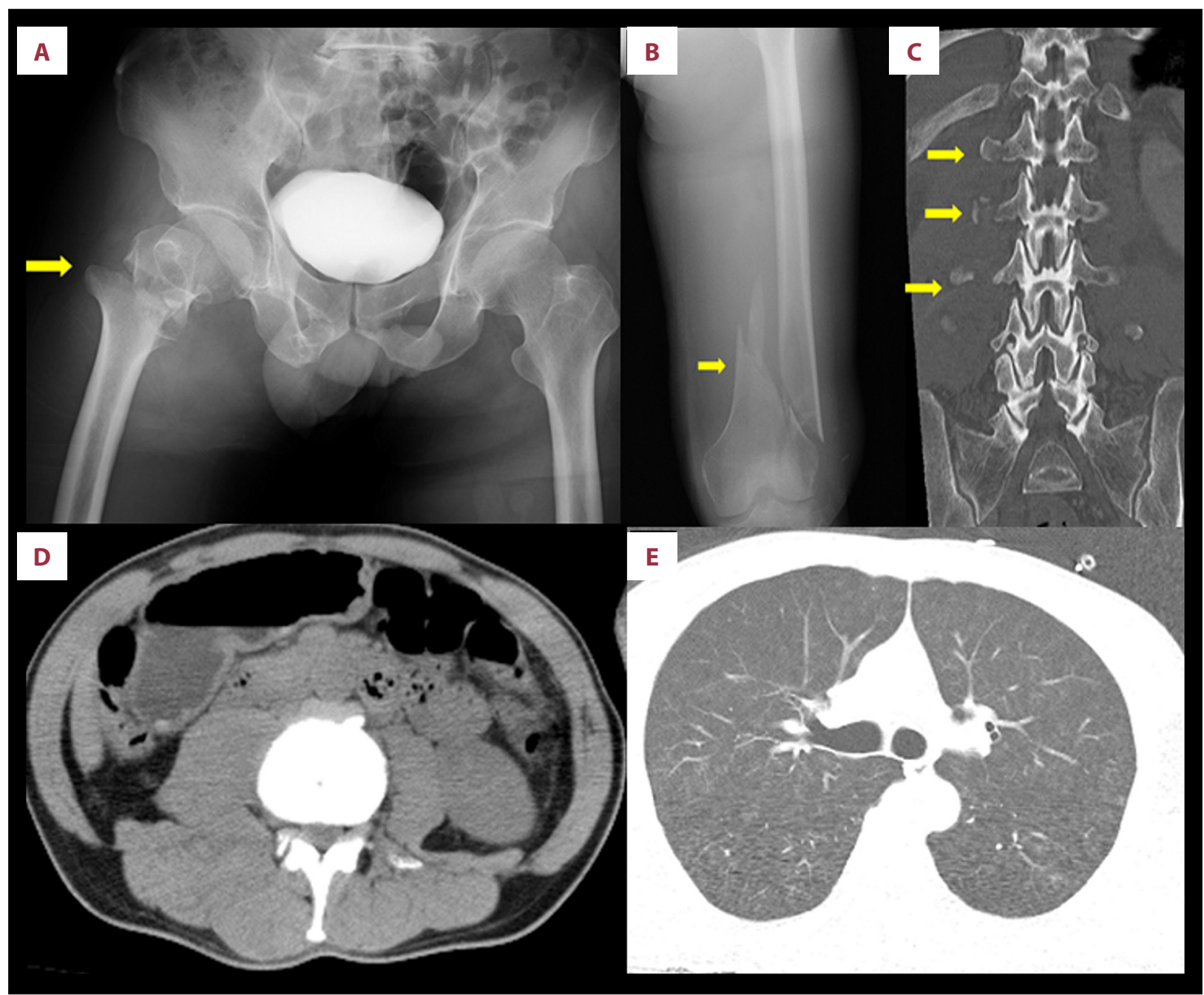

Figure 1. Radiographical findings of the major fractures and the lungs. Radiographs showing right trochanteric femoral fracture (A) and left distal femoral fracture (B). Computed tomography (CT) scans showing right lumbar transverse process fractures (C), and slight hemorrhage of right psoas major muscle by the right lumbar transverse process fracture, (D), and no findings of pneumonitis (E).

and the patient also underwent emergency angiography. We did not detect any pulmonary emboli. We could not perform coronary angiography because of unstable hemodynamics after immediate introduction of PCPS. We could not resuscitate the patient and pronounced him dead. Blood test results after cardiopulmonary arrest revealed WBC count, $1.75 \times 10^{3} / \mu \mathrm{L}$ with $78.8 \%$ neutrophils; $\mathrm{Hb}, 8.4 \mathrm{~g} / \mathrm{dL}$; Plt, $19.5 \times 10^{4} / \mu \mathrm{L}$; CRP, 54.10 mg/dL; TP, 4.2 g/dL; Alb 1.7 g/dL; AST, 346 U/L; ALT, 192 U/L; T.bil, $4.8 \mathrm{mg} / \mathrm{dL}$; CK, $7289 \mathrm{U} / \mathrm{L}$; creatine kinase-MB isoenzymes (CK-MB), 66 U/L; BUN, 39 mg/dL; Cre, $2.80 \mathrm{mg} / \mathrm{dL}$; PT-INR, 1.55; Fib, $832 \mathrm{mg} / \mathrm{dL}$; D-dimer, $18.5 \mu \mathrm{g} / \mathrm{mL}$. After his death on the $8^{\text {th }}$ day of hospitalization, results from 2 sets of blood cultures performed on the $5^{\text {th }}$ day revealed the growth of $C$. violaceum. Further analyses for antibiotic sensitivity revealed that this strain of $C$. violaceum was resistant to cephalosporins, aztreonam, sulbactam/ampicillin, and piperacillin/ tazobactam, but was sensitive to ciprofloxacin, levofloxacin, cefepime, and meropenem. Postmortem CT showed a bilateral pulmonary congestion and pleural effusion in addition to a low-density mass in the right psoas major muscle (Figure 3 ). Histopathological findings of the right psoas mass at autopsy revealed that the cause of the patient's death was sepsis caused by C. violaceum (Figure 4).

\section{Discussion}

Since the first reported case of human infection of $C$. violaceum from Malaysia in 1927, approximately over 200 cases of human $C$. violaceum infection have been reported worldwide $[1,7]$. Currently, only 6 cases from 5 case reports have been presented from Japan, and here we report the first case 


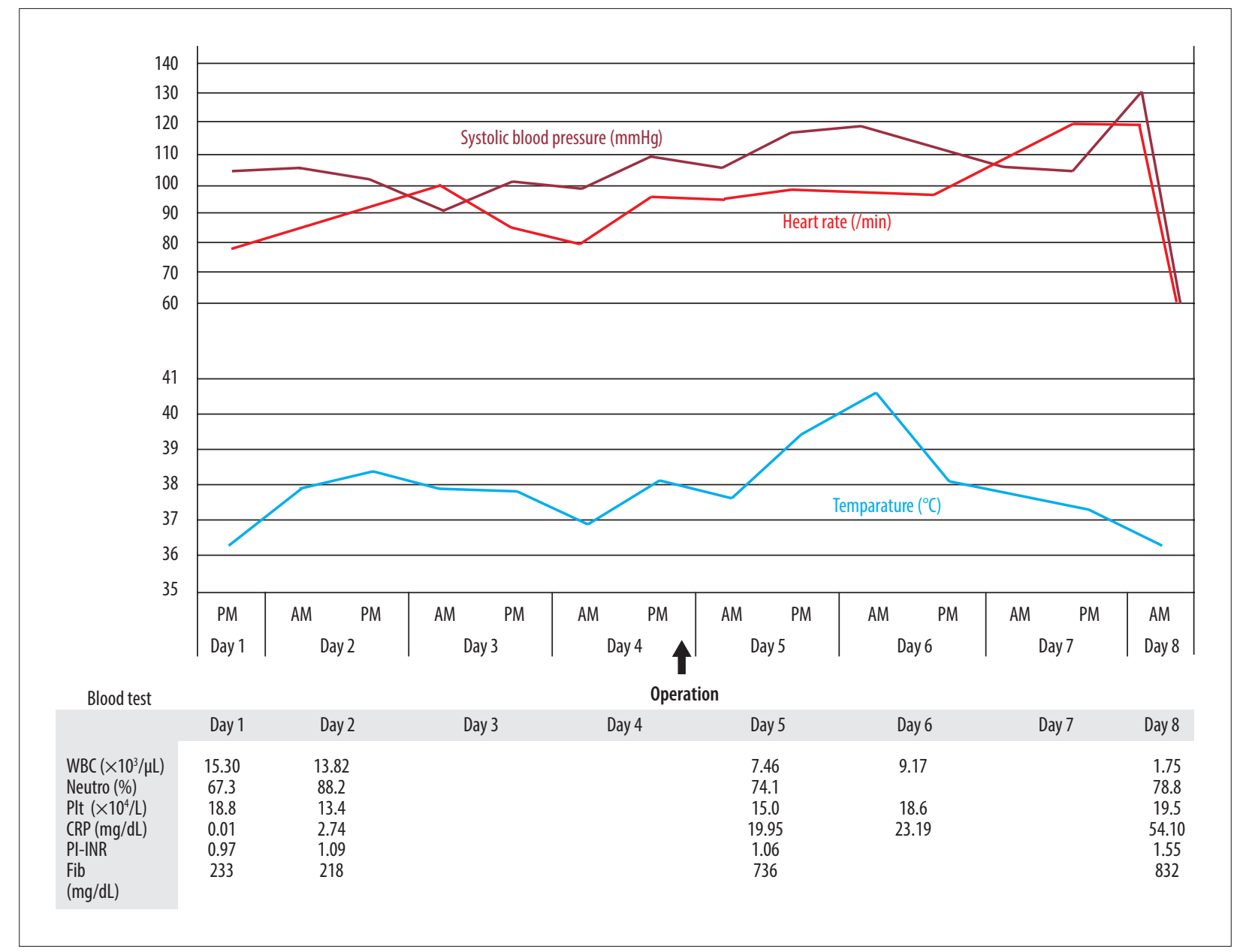

Figure 2. Temporal changes in major vital signs during the hospitalization and laboratory results. Plots showing changes in systolic blood pressure, heart rate, and temperature of the patient twice a day (AM and PM) on each day after hospital admission. WBC - white blood cell; Neutro - neutrophils; Plt - platelet; CRP - C-reactive protein; PT-INR - prothrombin timeinternational normalized ratio; Fib - fibrinogen.

of death caused by $C$. violaceum infection in Japan [2-3,8-10]. In East Asian countries with similar climate conditions, some fatal cases have been reported [11,12].

Since there are few reports of $C$. violaceum infection in Japan, most clinicians are unfamiliar with this infection, although the mortality rate of $C$. violaceum infection exceeds $50 \%$ and $C$. violaceum is endemic to approximately half of the geographical area of Japan (confined between latitudes of $35^{\circ} \mathrm{N}$ and $35^{\circ} \mathrm{S}$ ) [1]. Since there are only a few cases reported from Japan and nearly half of the country is endemic to $C$. violaceum, providers must maintain an index of suspicion of this pathogen in clinically relevant cases.

In the present case, $C$. violaceum infection progressed rapidly, and the patient died on the $8^{\text {th }}$ day of hospitalization, which was consistent with a previous study stating that the median survival duration of this infection was 7 days [1]. C. violaceum bacteremia progresses rapidly to fatal sepsis on dissemination to multiple organs, predominantly in the lungs, liver, and spleen, within a short time $[10,13,14]$. Nearly half of the patients had localized abscesses in visceral organs [1]. Cases presenting with both localized and iliopsoas abscesses are rare; only 1 of 106 patients presented with both localized and iliopsoas abscesses [1].

Trauma followed by exposure to contaminated water or soil is an important predisposing factor associated with this disease, even without swallowing the water and/or soil [1]. Traumainduced soft tissue infections, such as phlegmon, necrotizing fasciitis, and subcutaneous abscess, may also underlie $C$. violaceum infection [10]. The present case study underscores the importance of history-taking about exposure to contaminated water or soil even though patients denied a loss of consciousness or drawing and/or swallowing contaminated water or soil during episodes. Once exposure is confirmed, it is 


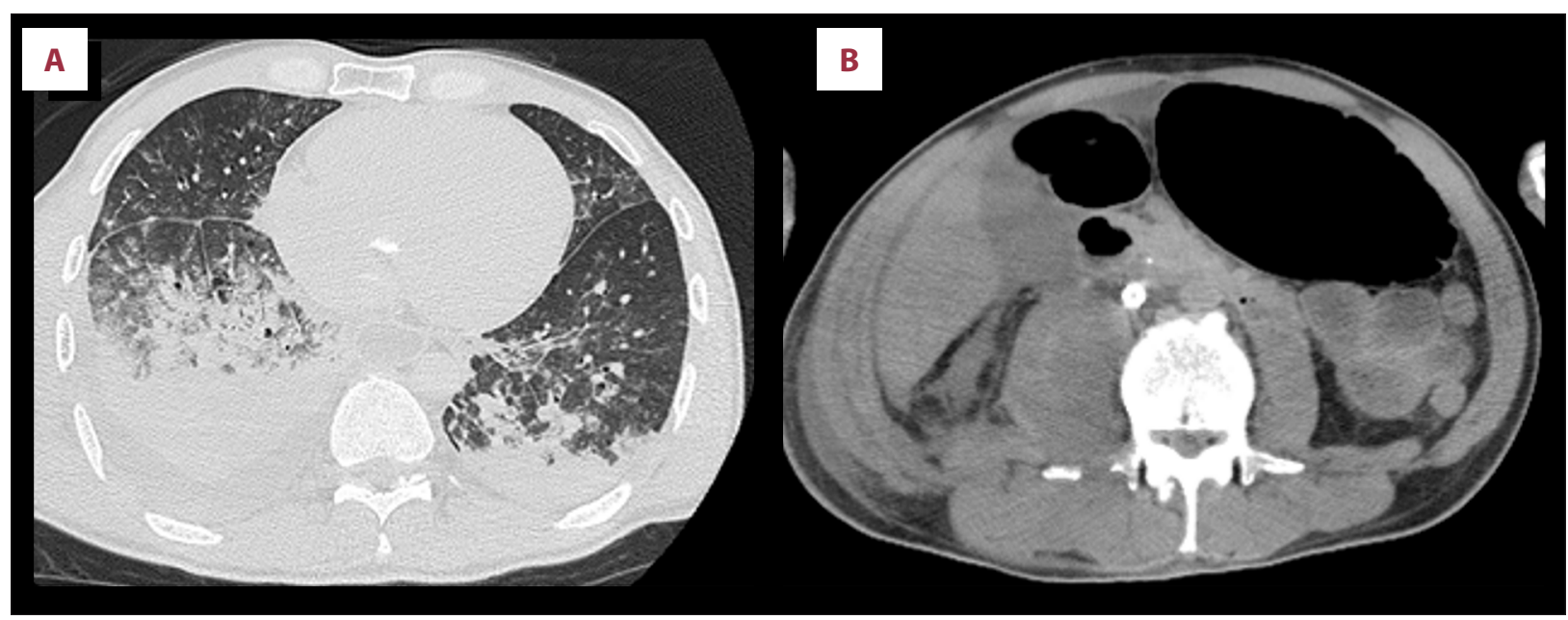

Figure 3. Postmortem computed tomography findings of the chest and abdomen. (A) Bilateral dorsal pulmonary congestion.

(B) Heterogeneous low-density mass in the right psoas muscle.

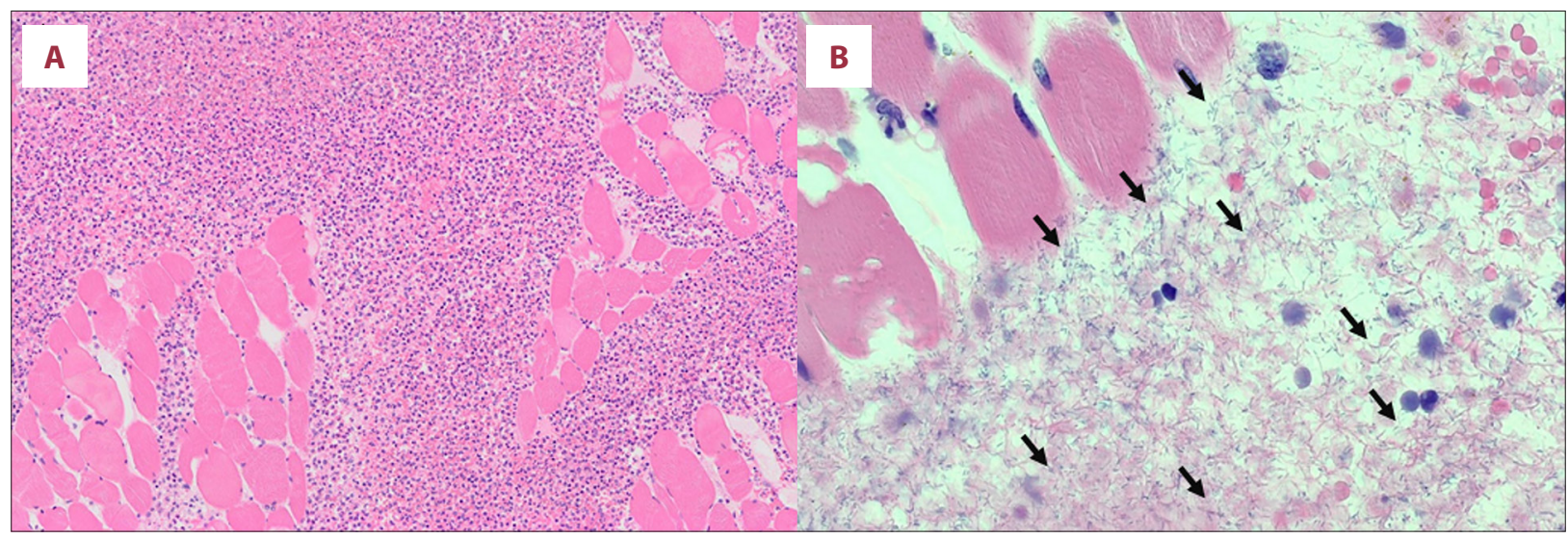

Figure 4. Microscopic findings of the psoas abscess at autopsy. (A) Light micrograph with hematoxylin and eosin staining showing psoas abscess and inflammatory cell infiltration by neutrophils and mononuclear cells. (B) At a higher magnification, numerous bacilli (arrows) are scattered.

necessary to include $C$. violaceum infection as a differential diagnosis of fever after trauma.

It was difficult to detect the infection owing to the lack of knowledge of $C$. violaceum. Multiple fractures were more likely causative of the patient's constant backache and lumbago. Hence, we did not suspect an abscess in the psoas major muscle. Moreover, treatment with NSAIDs for pain and postoperative fever masked his actual body temperature. Therefore, fever over $40^{\circ} \mathrm{C}$ after the surgery was the only clue of a serious infection. However, respiratory rate is an important indicator for sepsis, and we might have detected the patient's abnormality earlier [15]. It is conceivable that recording of respiratory rate as a one of the vital signs is essential in daily clinical practice.

As $C$. violaceum sepsis rarely occurs, little is known about its optimal antibiotic therapy. Most strains appear to be resistant to penicillin and cephalosporins [2-3,5]. In vitro studies showed that ciprofloxacin was the most effective antibiotic against $C$. violaceum $[2-3,5]$. As cefotiam was used for perioperative antibiotic treatment to prevent surgical site infection, it had no protective effect against $C$. violaceum infections. With global warming, C. violaceum infection has increased in nontropical climate regions, and several recent infections have occurred in geographical areas beyond those endemic to $C$. violaceum [1-3]. It is important to further study the multidrug resistance of $C$. violaceum and to choose broad-spectrum antibiotics such as ciprofloxacin.

\section{Conclusions}

In conclusion, we experienced the first fatal case of $C$. violaceum infection, in a 49-year-old man with no remarkable past medical history who died after a traffic accident. Exposure to contaminated water or soil, especially in summer, was considered 
as an important predisposing factor associated with C. violaceum infections. As most strains are resistant to penicillin and cephalosporins (including cefotiam of our case), ciprofloxacin may be the most effective antibiotic against $C$. violaceum. Because of the high mortality rate associated with this infection, it is vital to include $C$. violaceum infections as a differential diagnosis, since $C$. violaceum infections have increased in non-tropical counties.

\section{References:}

1. Yang CH, Li YH. Chromobacterium violaceum infection: A clinical review of an important but neglected infection. J Chin Med Assoc. 2011;74:435-41

2. Hagiya H, Murase T, Suzuki M, et al. Chromobacterium violaceum nosocomial pneumonia in two Japanese patients at an intensive care unit. J Infect Chemother. 2014;20:139-42

3. Matsuura N, Miyoshi M, Doi N, et al. Multiple liver abscesses with a skin pustule due to Chromobacterium violaceum. Intern Med. 2017;56:2519-22

4. Cheong BM. A fatal case of pulmonary Chromobacterium violaceum infection in an adult. Med J Malaysia. 2010;65:148-49

5. Moore CC, Lane JE, Stephens JL. Successful treatment of an infant with Chromobacterium violaceum sepsis. Clin Infect Dis. 2001;32:e107-10

6. Panchal AR, Bartos JA, Cabañas JG, et al. Part 3: Adult basic and advanced life support: 2020 American Heart Association Guidelines for Cardiopulmonary Resuscitation and Emergency Cardiovascular Care. Circulation. 2020;142(16 Suppl. 2):S366-468

7. Meher-Homji Z, Mangalore RP, Johnson PDR, Chua KYL. Chromobacterium violaceum infection in chronic granulomatous disease: A case report and review of the literature. JMM Case Rep. 2017;4(1):e005084

8. Hiraoka N, Yoshioka K, Inoue K, et al. Chromobacterium violaceum sepsis accompanied by bacteria-associated hemophagocytic syndrome in a Japanese man. Arch Intern Med. 1999;159:1623-24

\section{Acknowledgments}

We thank Ichiro Takeda for providing advice.

\section{Declaration of Figures Authenticity}

All figures submitted have been created by the authors who confirm that the images are original with no duplication and have not been previously published in whole or in part.

9. Nakamura A, Kojo Y, Nakagawa K. A case of refractory skin ulcer that occurred by Chromobacterium violaceum infection. The Nishinihon Journal of Dermatology. 2004;66:261-65. [in Japanese, Abstract in English]

10. Omori K, Jitsuiki K, Ohsaka H, et al. A patient with resistant Chromobacter iumviolaceum infection. Sch J Med Case Rep. 2015;3(8A):785-88

11. Ma SK, Chuang SK, Cheung TL, et al. A fatal case of Chromobacterium violaceum septicemia in Hong Kong. Southeast Asian J Trop Med Public Health. 2006;37(6):1179-82

12. Chang CY, Lee YT, Liu KS, et al. Chromobacterium violaceum infection in Taiwan: A case report and literature review. J Microbiol Immunol Infect. 2007;40(3):272-75

13. Martinez P, Mattar S. Fatal septicemia caused by Chromobacterium violaceum in a child from Colombia. Revista do Instituto de Medicina Tropical de São Paulo. 2007;49:391-93

14. Al Khalifa SM, Al Khaldi T, Alqahtani MM, Al Ansari AM. Two siblings with fatal Chromobacterium violaceum sepsis linked to drinking water. BMJ Case Rep. 2015;2015:bcr2015210987

15. Singer M, Deutschman CS, Seymour CW, et al. The third international consensus definitions for sepsis and septic shock (Sepsis-3) JAMA 2016;315(8):801-10 